\title{
Self-Calibration of Turntable Sequences from Silhouettes
}

\author{
Hui Zhang, Member, IEEE, and Kwan-Yee K. Wong, Member, IEEE
}

\begin{abstract}
This paper addresses the problem of recovering both the intrinsic and extrinsic parameters of a camera from the silhouettes of an object in a turntable sequence. Previous silhouette-based approaches have exploited correspondences induced by epipolar tangents to estimate the image invariants under turntable motion and achieved a weak calibration of the cameras. In order to recover the rotation angles and obtain a euclidean reconstruction, these approaches require the prior knowledge of the camera intrinsics. In this paper, we propose a novel approach for recovering the rotation angles precisely in the absence of the camera intrinsics. It is known that the fundamental matrix relating any two views in a turntable sequence can be expressed explicitly in terms of the image invariants, the rotation angle, and a fixed scalar. It will be shown that the imaged circular points for the turntable plane can also be formulated in terms of the same image invariants and fixed scalar. This allows the imaged circular points to be recovered directly from the estimated image invariants. The imaged circular points and the image invariants provide constraints for the estimation of the imaged absolute conic, and the camera calibration matrix can thus be recovered. A robust method for estimating the fixed scalar from image triplets is introduced, and a method for recovering the rotation angles by using the estimated imaged circular points and epipoles is presented. Using the estimated camera intrinsics and extrinsics, a euclidean reconstruction can be obtained. Experimental results on real data sequences are presented which demonstrate the high precision achieved by the proposed method.
\end{abstract}

Index Terms-Self-calibration, circular points, silhouettes, turntable motion.

\section{INTRODUCTION}

$\mathrm{T}$ HREE-DIMENSIONAL object reconstruction from image sequences has always been a hot research topic in both computer vision and computer graphics. Due to its ease of operation, a turntable has commonly been used with a fixed camera for acquiring images around an object in the modeling process. The most important and difficult step in object reconstruction from a turntable sequence is the recovery of the relative pose of the camera, the camera intrinsic parameters, and the rotation angles. Traditional approaches accomplish this task by attaching some special calibration patterns to the turntable to calibrate the camera [1], [2]. In [3], Fitzgibbon et al. introduced a point-based method for handling the case with an uncalibrated camera and unknown rotation angles. Their method is based on the projective geometry of single axis motion and it involves the computation of both fundamental matrices and trifocal tensors from point correspondences. Jiang et al. [4], [5] further extended this approach by making use of the conic trajectories of the rotating point features, and developed an algorithm that requires neither the computation of fundamental matrices nor trifocal tensors. Note that all of the

- H. Zhang is with the Division of Science and Technology, United International College, 28 Jingeng Road, Tangiiawan, Zhuhai, Guangdong, P.R. China, 519 085. E-mail: amyzhang@uic.edu.hk.

- K.-Y.K. Wong is with the Department of Computer Science, The University of Hong Kong, Pokfulam Road, Hong Kong.

E-mail:kykwong@cs.hku.hk.

Manuscript received 6 June 2007; revised 14 Jan. 2008; accepted 19 Feb. 2008; published online 10 Mar. 2008.

Recommended for acceptance by M. Polleyfeys.

For information on obtaining reprints of this article, please send e-mail to: tpami@computer.org, and reference IEEECS Log Number

TPAMI-2007-06-0334.

Digital Object Identifier no. 10.1109/TPAMI.2008.56. above approaches require the matching of point correspondences across the sequence, and would therefore not be applicable to smooth objects with sparse surface textures.

For smooth objects like sculptures, silhouettes are the predominant stable image features. Silhouettes are the projections of contour generators [6] which are viewpoint dependent. For two distinct viewpoints, the contour generators of an object are, in general, two distinct space curves. Hence, they provide no point correspondences between the corresponding silhouettes, except for the frontier points [7], which are the points of intersection between the two contour generators. Silhouette-based methods for motion estimation generally exploit epipolar tangents [8], [9], [10], [11] to locate the images of the frontier points to derive point correspondences between images. However, such an approach requires the presence of at least seven pairs of epipolar tangents, which may not be always available for objects with a simple shape. Besides, the location of epipolar tangents in a pair of uncalibrated images involves a nonlinear optimization with a nontrivial initialization. In [12], Sinha et al. proposed avoiding the problem of insufficient frontier points by using silhouettes of a dynamic object, and they also proposed a RANSAC approach for initializing the nonlinear optimization in locating the epipolar tangents. Nonetheless, such a method is not suitable for a static object.

In [13], Mendonça et al. developed a multistep algorithm for recovering the turntable motion. The number of required epipolar tangents per image pair is only two, which can be easily satisfied in almost all situations. By exploiting the symmetry properties exhibited in the image of a surface of revolution (SoR) generated by the rotating object, a simple method for locating epipolar tangents based on a one-dimensional search is introduced. Note that this 
method requires a dense turntable sequence in order to approximate the image of the SoR by the envelope of the silhouettes. In [14], Wong and Cipolla further extended the work in [13] to handle the case of sparse sequences, and their method also allows the incorporation of general views. The trade-off is the involvement of a high-dimensional optimization. Note that, in both [13] and [14], the recovery of the rotation angles and, furthermore, the euclidean reconstruction still require the knowledge of the camera intrinsics. In [15], Hernández et al. considered the problem of recovering both the turntable motion and the camera focal length from silhouettes. Rather than using the epipolar tangency constraint as in [13] and [14], they proposed a novel silhouette coherence constraint and developed a method that optimizes both the camera poses and the focal length simultaneously by maximizing the coherence between the silhouettes and the projections of the visual hull built from the silhouettes and the current estimate of the camera parameters. Like [14], their method involves a highdimensional optimization. It surpasses [14] by exploiting all the information contained in the contours of the silhouettes (rather than just the epipolar tangent points), and can handle incomplete silhouettes. Experimental results reported improvement over those obtained using epipolar tangents. In [16], Furukawa et al. proposed a RANSAC-based method for recovering general motion from silhouettes. They simplified the problem of locating potential epipolar tangents by restricting the viewing geometry to orthographic projection, under which all epipolar lines are parallel, and used signature representation of the dual image outlines to facilitate the matching of epipolar tangents. Although they also outlined how their method can be extended to handle the weak-perspective affine case, only results for the orthographic case were reported.

In this paper, we propose a novel algorithm for recovering both the intrinsic and extrinsic parameters of a camera from the silhouettes of an object in a turntable sequence. The proposed method follows the same approach as in [13], which first estimates the image invariants by using epipolar tangents and recovers the fundamental matrices in terms of these invariants. Based on the fact that the epipoles in one view are the images of the other camera centers, a simple method is proposed for precise estimation of the image invariants and rotation angles in the absence of the camera intrinsics. Such a method can also handle the degenerate case as described in [17] and [18], where the baseline passes through the scene object. Besides, it will be shown that the imaged circular points for the turntable plane can be obtained directly in terms of the image invariants and a fixed scalar used in the formula of the fundamental matrix. This allows the imaged circular points to be recovered directly from the estimated image invariants. The imaged circular points, together with the image invariants, provide constraints for the estimation of the imaged absolute conic, and the camera calibration matrix can thus be recovered. This can be used to upgrade the weakly calibrated cameras to fully calibrated ones, and a euclidean reconstruction follows. Preliminary results of this work have been published in [19].

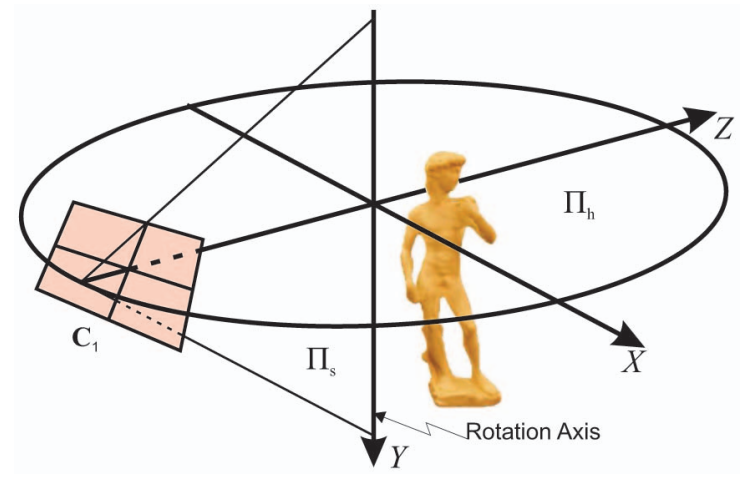

Fig. 1. Turntable motion. The reference camera $\mathbf{C}_{1}$ is initially lying on the negative $Z$-axis of the world coordinate system and it rotates about the $Y$-axis. The plane $\Pi_{\mathrm{h}}$ is defined by the positions of the rotating camera center, and the plane $\Pi_{\mathrm{s}}$ is defined by the $Y$-axis and the camera center.

The work presented here is most closely related to the work of Hernández et al. [15]. In [15], the authors considered the recovery of the focal length, which was estimated simultaneously with other motion parameters via an optimization in a high-dimensional space. In this work, we consider recovering three camera intrinsic parameters, namely, the focal length and the coordinates of the principal point. We also decouple the estimation of the camera intrinsic parameters from the extrinsic parameters and demonstrate that the intrinsic parameters can be recovered from the image invariants derived directly from the estimated motion parameters. This avoids potential problems of local minima often encountered in high-dimensional optimizations.

The remainder of this paper is organized as follows: Section 2 rehashes the image invariants under turntable motion and a special parameterization of the fundamental matrix in terms of the image invariants. Section 3 briefly reviews two existing algorithms for recovering the turntable motion from silhouettes upon which the algorithm proposed in this paper is based. Section 4 derives a novel formula for the imaged circular points expressed in terms of the image invariants and a fixed scalar, and presents an algorithm for recovering the imaged circular points from the estimated fundamental matrices. Besides, a robust method for computing the rotation angles, in the absence of the camera intrinsics, is also introduced. Section 5 shows the experimental results, followed by the conclusions in Section 6.

\section{Theoretical Background}

The geometry of a stationary camera viewing an object on a rotating turntable is equivalent to that of a camera rotating about the same axis and viewing a stationary object. For the sake of clear notations, we will refer to the geometry of the latter in the rest of this paper.

\subsection{Image Invariants under Turntable Motion}

Consider a reference camera $\mathbf{C}_{1}$ lying on the negative $Z$-axis of a world coordinate system and rotating about the $Y$-axis (see Fig. 1). The relative positions of the camera center describe a circle on a plane $\Pi_{\mathrm{h}}$ orthogonal to the rotation axis. The image of $\Pi_{h}$ is the line $l_{h}$, which is the vanishing 


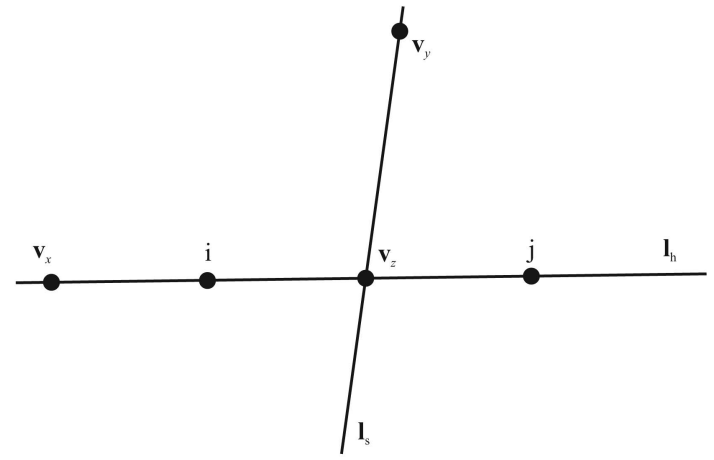

Fig. 2. Image invariants under turntable motion. The lines $\mathrm{l}_{\mathrm{h}}$ and $\mathrm{l}_{\mathrm{s}}$ are the images of $\Pi_{\mathrm{h}}$ and $\Pi_{\mathrm{s}}$, respectively. The points $\mathbf{v}_{x}, \mathbf{v}_{y}$, and $\mathbf{v}_{z}$ are the vanishing points for the normal direction of $\Pi_{s}$, the normal direction of $\Pi_{h}$, and a direction orthogonal to both the former two directions, respectively. The points $i$ and $\mathbf{j}$ are the imaged circular points for $\Pi_{h}$.

line (i.e., the horizon) for the turntable plane (see Fig. 2). Since all of the camera center positions lie on $\Pi_{\mathrm{h}}$, all of the epipoles $\mathbf{e}_{i j}$ that are images of the rotating camera center must lie on $l_{h}$, i.e.,

$$
\mathbf{l}_{\mathrm{h}}^{\mathrm{T}} \mathbf{e}_{i j}=0 \forall i, j .
$$

Let the plane defined by the camera center and the rotation axis be $\Pi_{\mathrm{s}}$, and consider three orthogonal directions, $\mathbf{N}_{x}, \mathbf{N}_{y}$, and $\mathbf{N}_{z}$, given by the normal direction of $\boldsymbol{\Pi}_{\mathbf{s}}$, the $Y$-axis, and $\mathbf{N}_{x} \times \mathbf{N}_{y}$, respectively (see Fig. 1). These three directions will have vanishing points $\mathbf{v}_{x}, \mathbf{v}_{y}$, and $\mathbf{v}_{z}$, respectively. The image of $\Pi_{\mathrm{s}}$ is $\mathbf{l}_{\mathrm{s}}$, which is also the image of the rotation axis (see Fig. 2). Since $\mathbf{N}_{x}$ is orthogonal to $\boldsymbol{\Pi}_{\mathrm{s}}$, it follows that $\mathbf{v}_{x}$ and $\mathbf{l}_{\mathrm{s}}$ form a pole-polar relationship with respect to the image of the absolute conic (IAC) $\omega$ [20], i.e.,

$$
\mathrm{l}_{\mathrm{s}} \sim \omega \mathbf{v}_{x} .
$$

Similarly, $\mathbf{N}_{y}$ is orthogonal to $\boldsymbol{\Pi}_{\mathrm{h}}$, and $\mathbf{v}_{y}$ and $\mathbf{l}_{\mathrm{h}}$ also form a pole-polar relationship with respect to the IAC $\omega$, i.e.,

$$
\mathrm{l}_{\mathrm{h}} \sim \omega \mathbf{v}_{y} .
$$

By construction, $\mathbf{N}_{x}$ is parallel to $\boldsymbol{\Pi}_{\mathrm{h}}, \mathbf{N}_{y}$ is parallel to $\boldsymbol{\Pi}_{\mathrm{s}}$, and $\mathbf{N}_{z}$ is parallel to both $\boldsymbol{\Pi}_{\mathrm{h}}$ and $\boldsymbol{\Pi}_{\mathrm{s}}$. Hence, $\mathbf{v}_{x}$ and $\mathbf{v}_{y}$ must lie on $l_{h}$ and $l_{\mathrm{s}}$, respectively, and $\mathbf{v}_{z}$ must lie on both $l_{h}$ and $1_{s}$, i.e.,

$$
\begin{gathered}
\mathbf{l}_{\mathrm{h}}^{\mathrm{T}} \mathbf{v}_{x}=0, \\
\mathbf{l}_{\mathrm{s}}^{\mathrm{T}} \mathbf{v}_{y}=0, \text { and } \\
\mathbf{v}_{z} \sim \mathbf{l}_{\mathrm{h}} \times \mathbf{l}_{\mathrm{s}} .
\end{gathered}
$$

Every circle on a plane will intersect the line at infinity at the circular points with canonical coordinates $\mathbf{I}=[1, j, 0]^{\mathrm{T}}$ and $\mathbf{J}=[1,-j, 0]^{\mathrm{T}}$ (where $j^{2}=-1$ ) [21]. The coordinates of these two conjugate complex points are invariant (as a pair) under any similarity transformation. The pair of imaged circular points $\mathbf{i}$ and $\mathbf{j}$ for the plane $\Pi_{\mathrm{h}}$ must lie on its vanishing line $\mathrm{l}_{\mathrm{h}}$, i.e.,

$$
\mathbf{l}_{\mathrm{h}} \sim \mathbf{i} \times \mathbf{j}
$$

Since a plane will intersect the absolute conic at the two circular points, it follows that the imaged circular points will lie on the IAC, i.e.,

$$
\mathbf{i}^{\mathrm{T}} \omega \mathbf{i}=\mathbf{j}^{\mathrm{T}} \omega \mathbf{j}=0 .
$$

If the intrinsic parameters of the camera are kept constant, due to symmetry in the configuration, the aforementioned vanishing points $\left(\mathbf{v}_{x}, \mathbf{v}_{y}\right.$, and $\left.\mathbf{v}_{z}\right)$, vanishing lines $\left(\mathbf{l}_{\mathrm{h}}\right.$ and $\left.\mathbf{l}_{\mathrm{s}}\right)$, and the imaged circular points ( $\mathbf{i}$ and $\mathbf{j}$ ) will remain unchanged throughout the sequence.

\subsection{Fundamental Matrix under Turntable Motion}

The fundamental matrix relating a view pair under turntable motion can be expressed explicitly in terms of the image invariants, and is given by [22], [3], [17]:

$$
\mathbf{F}_{i j}=\frac{1}{\operatorname{det}(\mathbf{K})}\left[\mathbf{v}_{x}\right]_{\times}+\tan \frac{\theta_{i j}}{2}\left(\mathbf{l}_{\mathrm{s}} \mathbf{l}_{\mathrm{h}}^{\mathrm{T}}+\mathbf{l}_{\mathrm{h}} \mathbf{l}_{\mathrm{s}}^{\mathrm{T}}\right),
$$

where $\mathbf{K}$ is the $3 \times 3$ calibration matrix of the camera and $\theta_{i j}$ is the rotation angle between the two views. Since $\mathbf{F}_{i j}$ is expressed as a sum of a skew-symmetric part and a symmetric part, the scales used in the homogeneous representations of the image invariants are important and cannot be ignored. In (9), $\mathbf{v}_{x}, \mathbf{l}_{\mathrm{s}}$, and $\mathbf{l}_{\mathrm{h}}$ must have the following forms:

$$
\begin{gathered}
\mathbf{v}_{x}=\mathbf{K R}\left[\begin{array}{lll}
1 & 0 & 0
\end{array}\right]^{\mathrm{T}}, \\
\mathbf{l}_{\mathrm{s}}=(\mathbf{K R})^{-\mathrm{T}}\left[\begin{array}{lll}
1 & 0 & 0
\end{array}\right]^{\mathrm{T}}, \text { and } \\
\mathbf{l}_{\mathrm{h}}=(\mathbf{K R})^{-\mathrm{T}}\left[\begin{array}{lll}
0 & 1 & 0
\end{array}\right]^{\mathrm{T}},
\end{gathered}
$$

where $\mathbf{R}$ is a $3 \times 3$ rotation matrix transforming vectors from the world coordinate system to the reference camera coordinate system. Given only the image invariants in an uncalibrated sequence (i.e., without the knowledge of $\mathbf{K}$ and $\mathbf{R}$ ), a scale factor $\kappa$ has to be introduced into the above formula to account for the different scales used in the homogeneous representations of the image invariants and the expression becomes

$$
\begin{aligned}
\mathbf{F}_{i j} & \sim\left[\mathbf{v}_{x}\right]_{\times}+\kappa \tan \frac{\theta_{i j}}{2}\left(\mathbf{l}_{\mathrm{s}} \mathbf{l}_{\mathrm{h}}^{\mathrm{T}}+\mathbf{l}_{\mathrm{h}} \mathbf{l}_{\mathrm{s}}^{\mathrm{T}}\right) \\
& \sim\left[\mathbf{v}_{x}\right]_{\times}+\lambda\left(\mathbf{l}_{\mathrm{s}} \mathbf{l}_{\mathrm{h}}^{\mathrm{T}}+\mathbf{l}_{\mathrm{h}} \mathbf{l}_{\mathrm{s}}^{\mathrm{T}}\right),
\end{aligned}
$$

where $\lambda=\kappa \tan \frac{\theta_{i j}}{2}$. If the scales used in the homogeneous representations of the image invariants are kept constant, the scale factor $\kappa$ will remain unchanged and will be independent of the particular view pair being considered. From (14), it is easy to see that a fundamental matrix relating a pair of views under turntable motion has only six degrees of freedom (DOF): two DOF for $l_{s}$, three DOF for $l_{h}$ and $\mathbf{v}_{x}$ (due to the constraint imposed by (4)), and one DOF for $\lambda$. If the image invariants have been recovered, only one DOF is needed to fix $\lambda$ and, hence, $\mathbf{F}_{i j}$. 


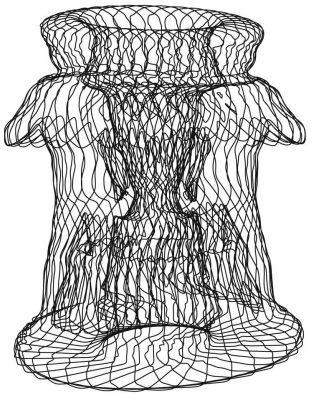

(a) (b)

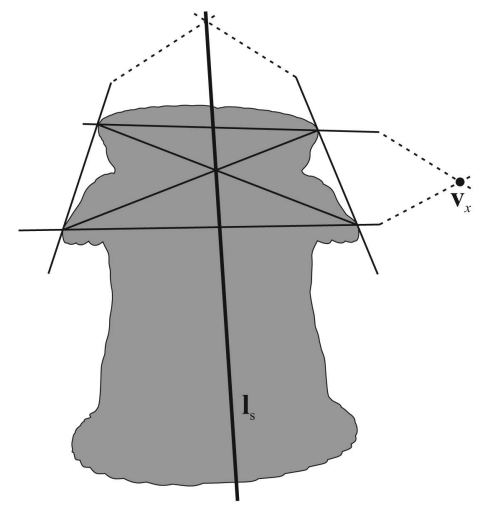

Fig. 3. (a) The image profile of the SoR generated by the rotating object can be approximated by the envelope of the deforming silhouettes of the object. (b) The image invariants $\mathbf{v}_{x}$ and $l_{\mathrm{s}}$ can be obtained from the intersections of the bitangent lines and lines formed from the bitangent points.

\section{Weak Calibration from Silhouettes}

In this section, two recent approaches for estimating turntable motion from silhouettes will be reviewed. Both methods estimate the fundamental matrices associated with the views under turntable motion by minimizing the symmetric transfer errors of the epipolar tangents to the silhouettes, and successful solutions have been developed by exploiting the special parameterization of the fundamental matrix. Note that, in order to recover the rotation angles between different views and achieve a euclidean reconstruction, the camera calibration matrix is needed in both methods to upgrade the weak calibration to a full calibration. In the next section, a novel method will be introduced to recover the camera calibration matrix from the estimated fundamental matrices. A robust method for recovering the rotation angles in the absence of the camera intrinsics will also be presented. These enhance the current algorithms by removing the requirement of known camera intrinsics, and allow a euclidean reconstruction from silhouettes in an uncalibrated turntable sequence.

\subsection{Dense Sequence}

Given a dense sequence, Mendonça et al. [17] introduced a multistep algorithm for estimating the turntable motion. Their method first recovers the imaged rotation axis $l_{\mathrm{s}}$ and the vanishing point $\mathbf{v}_{x}$ from the image profile $\rho$ of an SoR generated by the rotating object. They approximate such an image by the envelope of the deforming silhouettes. By exploiting the symmetry exhibited in $\rho, \mathbf{l}_{\mathbf{S}}$ and $\mathbf{v}_{x}$ can be easily obtained by locating bitangents to $\rho$ (see Fig. 3). Since $\rho$ is invariant to the transformation induced by a harmonic homology $\mathbf{W}$ with axis $\mathbf{l}_{\mathrm{s}}$ and vertex $\mathbf{v}_{x}$, defined as

$$
\mathbf{W}=\mathbf{I}-2 \frac{\mathbf{v}_{x} \mathbf{l}_{\mathrm{s}}^{\mathrm{T}}}{\mathbf{v}_{x}^{\mathrm{T}} \mathbf{l}_{\mathrm{s}}}
$$

$\mathbf{l}_{\mathrm{s}}$ and $\mathbf{v}_{x}$ can be further optimized by minimizing the transformation error of $\rho$ brought about by $\mathbf{W}$.

In the second step, the fact that corresponding epipolar lines are being mapped by $\mathbf{W}^{-\mathrm{T}}$ is exploited to locate the outer epipolar tangents to the silhouettes. The objective is to

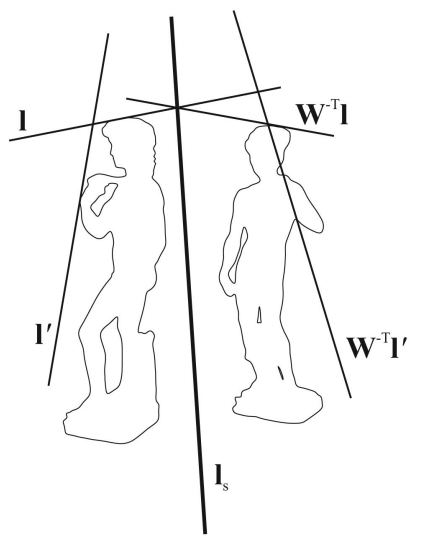

Fig. 4. An epipolar tangent is located by finding a line tangent to the silhouette in the first view, which is transformed by the harmonic homology $\mathbf{W}^{-\mathrm{T}}$ to a line tangent to the silhouette in the second view.

locate a line 1 tangent to the silhouette in one view, which will be transformed by $\mathbf{W}^{-\mathrm{T}}$ to a line $\mathbf{l}^{\prime}=\mathbf{W}^{-\mathrm{T}} \mathbf{l}$ tangent to the silhouette in the second view (see Fig. 4). This process can be carried out as a one-dimensional search in which the single search parameter is the angle that defines the orientation of the line 1 . The epipoles can then be obtained as the intersection point of the two outer epipolar tangents in each view, and the horizon $l_{h}$ is recovered as a line robustly fitted to the set of epipoles obtained from some randomly sampled image pairs (see Fig. 5).

After the first two steps, the image invariants $\mathbf{v}_{x}, \mathbf{l}_{\mathrm{s}}$, and $l_{h}$ have been recovered. The only missing term in the parameterization of the fundamental matrix $\mathbf{F}_{i j}$ given in (14) is $\lambda=\kappa \tan \frac{\theta_{i j}}{2}$. In the final step, a one-dimensional search along the parametric direction $\lambda$ is performed to optimize $\mathbf{F}_{i j}$ by minimizing the symmetric transfer errors of the epipolar tangents. After this optimization, the set of epipoles obtained from the resulting fundamental matrices will all lie on the horizon $\mathrm{l}_{\mathrm{h}}$.

With known camera intrinsics, the essential matrix can be formed from the fundamental matrix. The rotation angles of the camera can then be recovered by decomposing the essential matrices. A euclidean reconstruction can be obtained using the silhouettes and the set of projection matrices resulting from the decompositions.

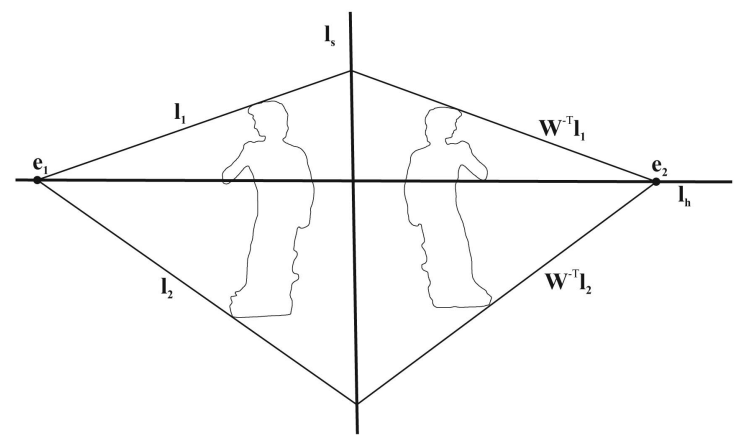

Fig. 5. The epipole is obtained as the intersection point of the two outer epipolar tangents in one view. The horizon $l_{h}$ is recovered as a line robustly fitted to the set of epipoles obtained from some randomly sampled image pairs. 


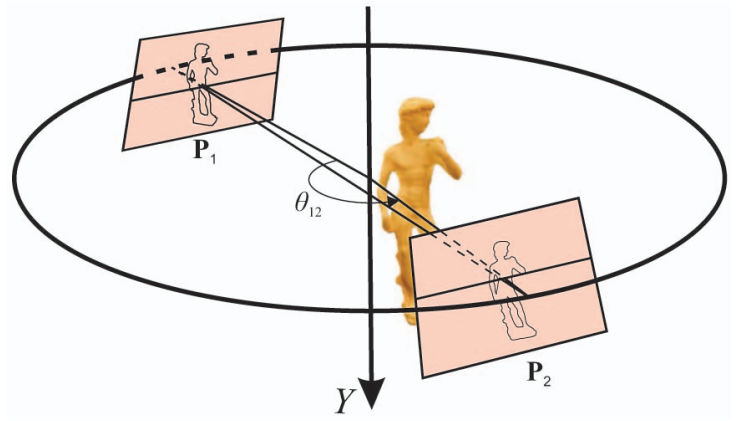

Fig. 6. In the degenerate case, the baseline passes through the object and outer epipolar tangents do not exist.

The advantages of the above approach are that it requires the presence of only two epipolar tangents per image pair, and involves only two one-dimensional searches with trivial initializations. Nonetheless, it requires a dense (say, with rotation angles less than $10^{\circ}$ ) and complete (i.e., performing a complete $360^{\circ}$ rotation) sequence for approximating the image of an SoR generated by the rotating object, and requires the prior knowledge of the camera calibration matrix to recover the rotation angles and achieve a euclidean reconstruction. Furthermore, the method will fail if the baseline of an image pair passes through the object. Under this degenerate case, the epipoles will be located inside the silhouettes and the location of the outer epipolar tangents becomes impossible (see Fig. 6).

\subsection{Sparse Sequence}

The method described in the previous section works on a dense complete turntable sequence. In [18], Wong and Cipolla considered the case of a sparse incomplete turntable sequence. Their method exploits the special parameterization of the fundamental matrix given in (13), and assumes that the camera calibration matrix $\mathbf{K}$ is known. Given the camera calibration matrix $\mathbf{K}$, the IAC can be recovered as $\omega \sim\left(\mathbf{K K}^{\mathrm{T}}\right)^{*}$, and $\mathbf{v}_{x}$ can be uniquely determined by $\mathbf{l}_{\mathrm{s}}$ by using (2). The horizon $\mathbf{l}_{\mathrm{h}}$ can be defined by $\mathbf{v}_{x}$ and its intersection $\mathbf{v}_{z}$ with $\mathbf{l}_{\mathrm{s}}$. Hence, $\mathbf{v}_{x}, \mathbf{l}_{\mathrm{s}}$, and $\mathbf{l}_{\mathrm{h}}$ altogether have only three DOF. Note that, with $\mathbf{v}_{x}, \mathbf{l}_{\mathrm{s}}$, and $\mathbf{l}_{\mathrm{h}}$ being properly normalized, the fixed scalar $\kappa$ can be determined from the determinant of $\mathbf{K}$. Given a sequence of $N$ images, there are $N-1$ rotation angles between adjacent views, and therefore a total of $3+(N-1)=N+2$ parameters are needed to describe all the fundamental matrices (i.e., motion parameters) associated with the sequence. The method in [18] begins by manually initializing $\mathbf{l}_{\mathrm{s}}$ (and hence $\mathbf{v}_{x}$ ) and selecting a point on $\mathrm{l}_{\mathrm{s}}$ to define $\mathrm{l}_{\mathrm{h}}$. The $N-1$ rotation angles between the viewpoints are arbitrarily initialized. These $N+2$ parameters are then optimized by minimizing the symmetric transfer errors of the epipolar tangents to the silhouettes. After the optimization, the fundamental matrices between adjacent viewpoints are formed, which are then upgraded to the essential matrices by using the camera calibration matrix. A set of projection matrices compatible with the turntable motion can then be obtained by decomposing the resulting essential matrices. In [18], Wong and Cipolla also proposed a method for registering new general views with the silhouettes in the turntable sequence, which optimizes the six extrinsic parameters of the camera by minimizing the symmetric transfer errors of the epipolar tangents. Interested readers are referred to [14] and [18] for details of this algorithm. Under the assumptions of unit aspect ratio and the principal point being at the image center, a minor modification, i.e., by including also the focal length $f$ in the optimization parameters (i.e., a total of $N+3$ parameters), will produce an algorithm similar to [15].

Compared with the dense sequence method, the above method can handle sparse and incomplete turntable sequences. However, it involves a nonlinear optimization in a high-dimensional $(N+2)$ space. Nonetheless, the fact that these $N+2$ parameters all have physical meanings (e.g., the image of the rotation axis, the horizon, and rotation angles) makes the initialization of the nonlinear optimization an easy task. Like the dense sequence method, this method requires prior knowledge of the camera calibration matrix to recover the rotation angles and achieve a euclidean reconstruction, and will fail under the previously described degenerate case.

\section{Self-Calibration of Turntable Motion}

In this section, a novel parameterization for the imaged circular points of the turntable plane will be introduced. This allows the imaged circular points to be recovered directly from the estimated image invariants. The imaged circular points thus obtained, together with other image invariants, will be exploited to develop a method for recovering the camera calibration matrix. Finally, a robust method for recovering the rotation angles in the absence of the camera intrinsics is presented. These extend existing methods for turntable motion estimation from silhouettes to handle uncalibrated sequences by removing the restrictive requirement of the prior knowledge of the camera intrinsics.

\subsection{Parameterization for the Imaged Circular Points}

In this section, a new formula for the imaged circular points of the turntable plane will be derived. It will be shown that the imaged circular points can be expressed in terms of the image invariants and the fixed scalar used in the special parameterization of the fundamental matrix for turntable sequence. The exact expression for the imaged circular points of the turntable plane in terms of the image invariants is given in Proposition 1.

Proposition 1. Given a turntable sequence, the imaged circular points $\mathbf{i}$ and $\mathbf{j}$ of the turntable plane can be expressed as

$$
\mathbf{i}, \mathbf{j} \sim \mathbf{v}_{x} \pm j \kappa\left(\mathbf{l}_{\mathrm{s}} \times \mathbf{l}_{\mathrm{h}}\right),
$$

where $j^{2}=-1$ and $\mathbf{v}_{x}, \mathbf{l}_{\mathrm{s}}, \mathbf{l}_{\mathrm{h}}$, and $\kappa$ are the image invariants and fixed scale factor used in the special parameterization of the fundamental matrix for turntable motion, as given in (13).

Proof. Without loss of generality, consider two views in the turntable sequence with projection matrices $\mathbf{P}_{1}=$ $\mathbf{K R}[\mathbf{I}-\mathbf{C}]$ and $\mathbf{P}_{2}=\mathbf{K R}\left[\mathbf{R}_{Y}\left(\theta_{12}\right)-\mathbf{C}\right]$, respectively, where $\mathbf{R}_{Y}\left(\theta_{12}\right)$ is a rotation about the $Y$-axis by an angle $\theta_{12}$ and $\mathbf{C}=\left[\begin{array}{lll}0 & 0 & -1\end{array}\right]^{\mathrm{T}}$ is the camera center (see Fig. 7). The image $\mathrm{x}$ of a point $\mathbf{X}=\left[\begin{array}{llll}X & 0 & Z & 1\end{array}\right]^{\mathrm{T}}$ on the $X-Z$ plane under $\mathbf{P}_{1}$ is given by 


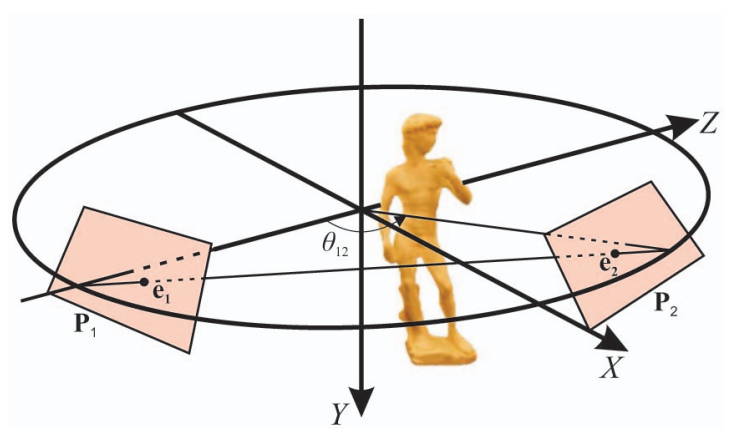

Fig. 7. A pair of cameras $\mathbf{P}_{1}$ and $\mathbf{P}_{2}$ related by a rotation with an angle $\theta_{12}$ about the $Y$-axis.

$$
\begin{aligned}
\mathbf{x} & \sim\left[\begin{array}{llll}
\mathbf{p}_{1} & \mathbf{p}_{2} & \mathbf{p}_{3} & \mathbf{p}_{4}
\end{array}\right]\left[\begin{array}{c}
X \\
0 \\
Z \\
1
\end{array}\right] \\
& =\left[\begin{array}{ll}
\mathbf{p}_{1} & \mathbf{p}_{3}
\end{array}\right]\left[\begin{array}{c}
X \\
Z+1
\end{array}\right],
\end{aligned}
$$

where $\mathbf{p}_{c}(c=1,2,3,4)$ are the columns of $\mathbf{P}_{1}$ and $\mathbf{p}_{3}=\mathbf{p}_{4}$. Since, under $\mathbf{P}_{1}, \mathbf{v}_{x}$ and $\mathbf{v}_{z}$ are the vanishing points of the $X$-axis and $Z$-axis, respectively, it follows that $\mathbf{p}_{1} \sim \mathbf{v}_{x}$ and $\mathbf{p}_{3} \sim \mathbf{v}_{z}$ [3]. The epipole $\mathbf{e}_{i}$ on the image of $\mathbf{P}_{i}$ is the projection of the point $\left[\begin{array}{llll}\sin \left(\pi+(-1)^{i} \theta_{12}\right) & 0 & \cos \left(\pi+(-1)^{i} \theta_{12}\right) & 1\end{array}\right]^{\mathrm{T}}$ and is given by

$$
\begin{aligned}
\mathbf{e}_{i} & \sim\left[\begin{array}{ll}
\mathbf{p}_{1} & \mathbf{p}_{3}
\end{array}\right]\left[\begin{array}{c}
\sin \left(\pi+(-1)^{i} \theta_{12}\right) \\
\cos \left(\pi+(-1)^{i} \theta_{12}\right)+1
\end{array}\right] \\
& \sim\left[\begin{array}{ll}
\mathbf{v}_{x} & \beta \mathbf{v}_{z}
\end{array}\right]\left[\begin{array}{c}
\sin \left(\pi+(-1)^{i} \theta_{12}\right) \\
\cos \left(\pi+(-1)^{i} \theta_{12}\right)+1
\end{array}\right] \\
& \sim\left[\begin{array}{ll}
\mathbf{v}_{x} & \beta\left(\mathbf{l}_{\mathrm{s}} \times \mathbf{l}_{\mathrm{h}}\right)
\end{array}\right]\left[\begin{array}{c}
\sin \left(\pi+(-1)^{i} \theta_{12}\right) \\
\cos \left(\pi+(-1)^{i} \theta_{12}\right)+1
\end{array}\right] \\
& \sim \mathbf{v}_{x}-(-1)^{i} \beta \tan \frac{\theta_{12}}{2}\left(\mathbf{l}_{\mathrm{s}} \times \mathbf{l}_{\mathrm{h}}\right),
\end{aligned}
$$

where $\beta$ is an unknown fixed scalar used to fix the relative scales between $\mathbf{v}_{x}$ and $\mathbf{v}_{z}$, and $\mathbf{v}_{z} \sim \mathbf{l}_{\mathrm{S}} \times \mathbf{l}_{\mathrm{h}}$ (see (6)). Note that $\mathbf{e}_{1}$ and $\mathbf{e}_{2}$ are the right and left null spaces of the fundamental matrix $\mathbf{F}_{12}$ relating the two views. From (13), $\mathbf{F}_{12}$ has the form

$$
\mathbf{F}_{12} \sim\left[\mathbf{v}_{x}\right]_{\times}+\kappa \tan \frac{\theta_{12}}{2}\left(\mathbf{l}_{\mathrm{s}} \mathbf{l}_{\mathrm{h}}^{\mathrm{T}}+\mathbf{l}_{\mathrm{h}} \mathbf{l}_{\mathrm{s}}^{\mathrm{T}}\right) .
$$

Solving $\mathbf{F}_{12} \mathbf{e}_{1}=\mathbf{0}$ and $\mathbf{F}_{12}^{\mathrm{T}} \mathbf{e}_{2}=\mathbf{0}$ gives $\beta=\kappa$. Finally, the imaged circular points $\mathbf{i}, \mathbf{j}$ can be obtained by projecting the circular points $\mathbf{I}, \mathbf{J}=\left[\begin{array}{llll}1 & 0 & \pm j & 0\end{array}\right]^{\mathrm{T}}$ under $\mathbf{P}_{1}$, i.e.,

$$
\begin{aligned}
\mathbf{i}, \mathbf{j} & \sim \mathbf{p}_{1} \pm j \mathbf{p}_{3} \\
& \sim \mathbf{v}_{x} \pm j \kappa\left(\mathbf{l}_{\mathrm{s}} \times \mathbf{l}_{\mathrm{h}}\right),
\end{aligned}
$$

and the proof is completed.

\subsection{Recovery of the Camera Calibration Matrix}

By exploiting the parameterization introduced in the previous section, the imaged circular points of the turntable plane can be recovered once the fundamental matrix has

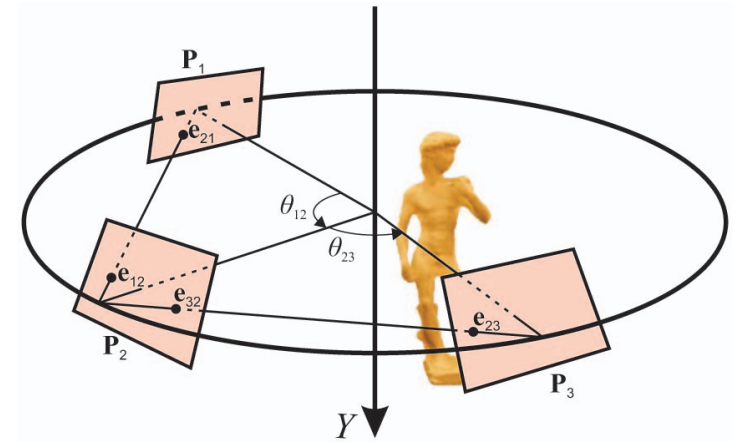

Fig. 8. Three views under turntable motion. Epipole $\mathbf{e}_{p q}$ is the projection of the camera center of view $p$ onto view $q . \theta_{p q}$ is the rotation angle between views $p$ and $q(p, q=1,2,3 p \neq q)$.

been estimated. In this work, the image invariants and the fundamental matrices are first estimated using the dense sequence method [17], as described in Section 3.1. The unknown scale factor $\kappa$ used in the formula of the imaged circular points can be recovered by considering a triplet of views. Let the three views have relative rotation angles $\theta_{p q}$, where $p, q=1,2,3, p \neq q$ (see Fig. 8), and $\lambda_{1}=\kappa \tan \frac{\theta_{12}}{2}$, $\lambda_{2}=\kappa \tan \frac{\theta_{23}}{2}$, and $\lambda_{3}=\kappa \tan \frac{\theta_{13}}{2}$, respectively. Using the fact that $\theta_{13}=\theta_{12}+\theta_{23}$, the fixed scalar $\kappa$ can be obtained as

$$
\kappa=\sqrt{\frac{\lambda_{1} \lambda_{2} \lambda_{3}}{\lambda_{3}-\lambda_{1}-\lambda_{2}}} .
$$

Due to the existence of noises, $\kappa$ estimated from different triplets may have different values. In this work, a set of $\kappa$ is recovered from some randomly sampled image triplets, and the mode is then chosen as the best estimate.

Since the imaged circular points lie on the IAC $\omega$,

$$
\mathbf{i}^{\mathrm{T}} \omega \mathbf{i}=\mathbf{j}^{\mathrm{T}} \omega \mathbf{j}=0 .
$$

Besides, the imaged rotation axis $\mathbf{l}_{\mathrm{S}}$ and the vanishing point $\mathbf{v}_{x}$ define a pole-polar relationship with respect to the IAC [21]. Hence,

$$
\mathbf{l}_{\mathrm{S}} \sim \omega \mathbf{v}_{x}
$$

Equations (21) and (22) together provide three independent constraints on $\omega$, which allow $\omega$ to be estimated under the assumption of a natural camera with zero skew and unit aspect ratio. Finally, the camera calibration matrix $\mathbf{K}$ can be obtained by the Cholesky decomposition [23] of $\omega$.

\subsection{Recovery of the Rotation Angles}

Having estimated the fixed scalar $\kappa$, a simple way of recovering the rotation angles is to divide the term $\lambda=$ $\kappa \tan (\theta / 2)$ in (14) by $\kappa$ [19]. However, such an approach cannot be applied to the degenerate case shown in Fig. 6 since the fundamental matrix (and hence $\lambda$ ) cannot be recovered in such a case. This problem can be avoided by acquiring the images in a way such that the horizon is above or below all the silhouettes. In this paper, this problem is solved by making use of the available epipoles from all image pairs.

Consider the top view of the turntable plane (i.e., the $X-Z$ plane; see Fig. 9). The reference camera $\mathbf{C}_{1}$ (lying on the negative $Z$-axis) rotates about the origin $\mathrm{O}$ and the 


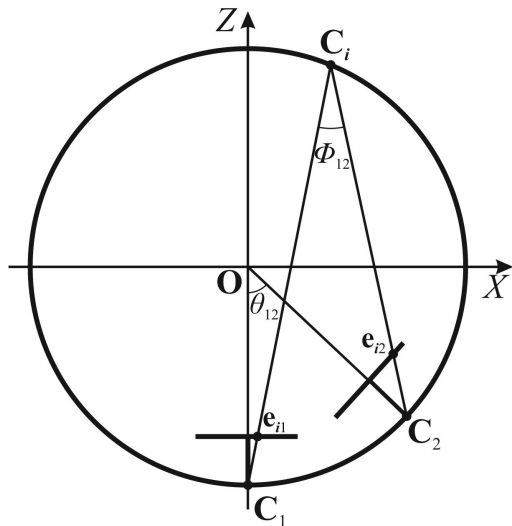

Fig. 9. Two views and one point configuration: The epipoles $\mathbf{e}_{i 1}$ and $\mathbf{e}_{i 2}$ are the projections of the camera center $\mathbf{C}_{i}$ in the images taken at $\mathbf{C}_{1}$ and $\mathbf{C}_{2}$, respectively, where $\mathbf{C}_{2}$ is obtained by rotating $\mathbf{C}_{1}$ about the origin by an angle $\theta_{12}$.

relative positions of the camera center $\mathbf{C}_{i}(i=1, \ldots, m)$ describe a circle on the $X-Z$ plane. Consider the camera center at two positions $\mathbf{C}_{1}$ and $\mathbf{C}_{2}$ with a relative rotation angle $\theta_{12}$, and a camera center at another position $\mathbf{C}_{i}$ where $i \neq 1,2$. The epipoles $\mathbf{e}_{i 1}$ and $\mathbf{e}_{i 2}$ are the projections of the camera center $\mathbf{C}_{i}$ in the images taken at $\mathbf{C}_{1}$ and $\mathbf{C}_{2}$, respectively. By rotating both $\mathbf{C}_{2}$ and $\mathbf{C}_{i}$ about the origin by an angle $\theta_{12}$ toward $\mathbf{C}_{1}, \mathbf{C}_{2}$ will coincide with $\mathbf{C}_{1}$ (see Fig. 10). Let the new position of $\mathbf{C}_{i}$ be $\mathbf{C}_{i}^{\prime}$. It is easy to see that the rotation angle between $\mathbf{C}_{i}$ and $\mathbf{C}_{i}^{\prime}$ is $\theta_{12}$, and $\mathbf{e}_{i 2}$ is equivalent to the projection of $\mathbf{C}_{i}^{\prime}$ in the image taken at $\mathbf{C}_{1}$ [24]. Let the viewing rays from $\mathbf{C}_{1}$ to $\mathbf{C}_{i}$ and $\mathbf{C}_{i}^{\prime}$ be $\mathbf{L}_{i 1}$ and $\mathbf{L}_{i 2}$, respectively. By simple trigonometry, the angle $\phi_{12}$ between $\mathbf{L}_{i 1}$ and $\mathbf{L}_{i 2}$ is equal to half the rotation angle $\theta_{12}$, i.e., $\phi_{12}=\frac{\theta_{12}}{2}$. The angle $\phi_{12}$ can be obtained using Laguerre's formula:

$$
\phi_{12}=\frac{1}{2 j} \log \left\{\mathbf{L}_{i 1}, \mathbf{L}_{i 2} ; \mathbf{L}_{\mathbf{I} 1}, \mathbf{L}_{\mathbf{J} 1}\right\},
$$

where $\left\{\mathbf{L}_{i 1}, \mathbf{L}_{i 2} ; \mathbf{L}_{\mathbf{I} 1}, \mathbf{L}_{\mathbf{J} 1}\right\}$ denotes a cross ratio, $j^{2}=-1$, and $\mathbf{L}_{\mathbf{I} 1}$ and $\mathbf{L}_{\mathbf{J} 1}$ are the isotropic lines through $\mathbf{C}_{1}$ (i.e., the lines from $\mathbf{C}_{1}$ to the circular points $\mathbf{I}$ and $\mathbf{J}$, respectively) [25].

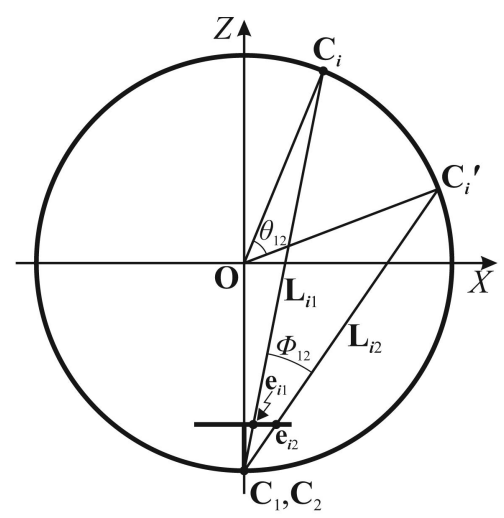

Fig. 10. Two points and one view configuration: By rotating both $\mathrm{C}_{2}$ and $\mathbf{C}_{i}$ about the origin by an angle $\theta_{12}$ toward $\mathbf{C}_{1}, \mathbf{C}_{2}$ will coincide with $\mathbf{C}_{1}$. Let the new position of $\mathbf{C}_{i}$ be $\mathbf{C}_{i}^{\prime}$. $\mathbf{e}_{i 2}$ can then be regarded as the projection of the camera center $\mathbf{C}_{i}^{\prime}$ in the image taken at $\mathbf{C}_{1}$.

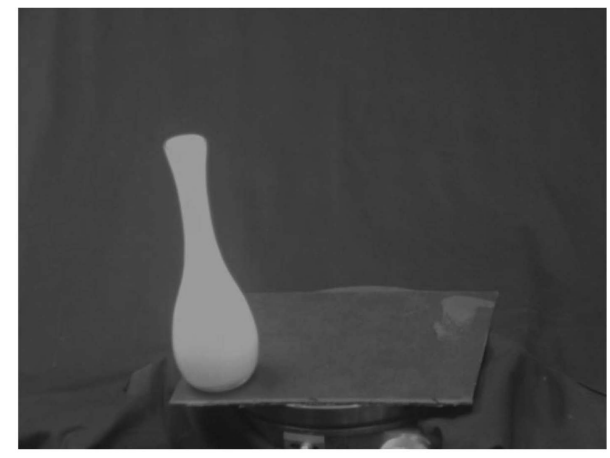

Fig. 11. An image from the vase sequence.

Given the epipoles $\left\{\mathbf{e}_{i 1}, \mathbf{e}_{i 2}\right\}$ and the imaged circular points $\{\mathbf{i}, \mathbf{j}\}, \phi_{12}$ can therefore be estimated as [4]

$$
\phi_{12}=\frac{1}{2 j} \log \left\{\mathbf{e}_{i 1}, \mathbf{e}_{i 2} ; \mathbf{i}, \mathbf{j}\right\},
$$

and the rotation angle $\theta_{12}$ follows. Note that the accuracy of the recovered angle $\theta_{12}$ depends on the accuracy of the epipoles $\mathbf{e}_{i 1}$ and $\mathbf{e}_{i 2}$ (i.e., the accuracy of the estimated fundamental matrices $\mathbf{F}_{1 i}$ and $\mathbf{F}_{2 i}$ ) being used. To handle the degenerate case robustly and obtain an accurate estimate of the rotation angle, all available image pairs are considered to compute a set of estimated angles and the median is then chosen as the robust estimate of $\theta_{12}$.

\section{Experimental Results}

The first experimental sequence consists of 72 images of a vase (see Fig. 11). The images have a resolution of $640 \times 480$. Each image was taken by sequentially rotating the object by $5^{\circ}$ on a manually operated turntable with a resolution of $0.01^{\circ}$. The silhouettes were extracted using cubic B-spline snakes [26], [27] with manual initializations. The image invariants $\mathbf{l}_{\mathrm{s}}, \mathbf{v}_{x}$, and $\mathbf{l}_{\mathrm{h}}$ were first estimated from the whole sequence by using the dense sequence method as described in Section 3.1. A subsequence consisting of 18 images with successive rotation angles of $20^{\circ}$ was then extracted for the euclidean reconstruction of the vase. The fixed scalar $\kappa$ and the rotation angles were estimated from this subsequence by using the methods described in Sections 4.2 and 4.3, respectively. All the parameters $\left(\mathbf{v}_{x}, \mathbf{l}_{\mathrm{s}}, \mathbf{l}_{\mathrm{h}}, \kappa\right.$, and rotation angles) were then optimized in a bundle adjustment, which minimized the symmetric transfer errors of the epipolar tangents to the silhouettes by using (13). The camera calibration matrix was then recovered from the optimized image invariants by using the method described in Section 4.2. Finally, a set of projection matrices compatible with the turntable motion was obtained from the decomposition of the essential matrices, and a euclidean reconstruction was achieved using a visual hull from silhouettes method as described in [28].

Fig. 12 shows the histogram of the estimated values for $\kappa$ from some randomly sampled image triplets, and the peak indicates the mode that was taken as the robust estimate of $\kappa$. Fig. 13 shows both the rotation angles obtained before and after the bundle adjustment. The root-mean-square (RMS) errors of the estimated rotation angles before and 


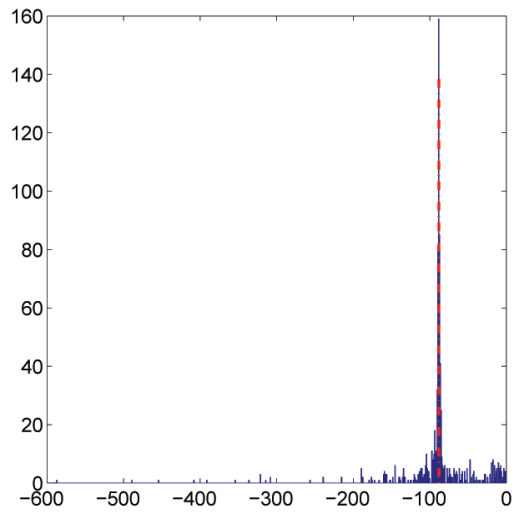

Fig. 12. Vase sequence: histogram of the estimated values for $\kappa$. The dashed line indicates the "best estimate" $\kappa_{0}$.

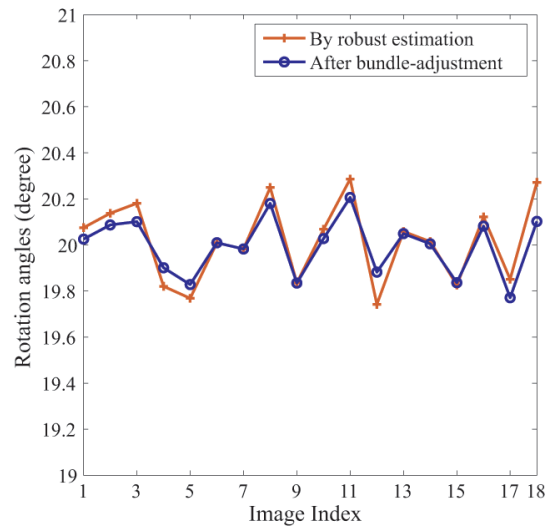

Fig. 13. Vase sequence: angles estimated using the robust method and those obtained after bundle adjustment. The RMS errors of the estimated rotation angles before and after the bundle adjustment are $0.134^{\circ}$ and $0.112^{\circ}$, respectively.

TABLE 1

Estimated and Ground-Truth Values for the Intrinsics of the Vase Sequence

\begin{tabular}{|c|ccc|}
\hline- & $f$ & $u_{0}$ & $v_{0}$ \\
\hline Ground-truth & 2389.8 & 342.83 & 255.32 \\
Estimated value & 2366.5 & 336.76 & 393.35 \\
Percentage error & $0.97 \%$ & $0.25 \%$ & $5.78 \%$ \\
\hline
\end{tabular}

after the bundle adjustment are $0.134^{\circ}$ and $0.112^{\circ}$, respectively, which are better than the RMS error of $0.192^{\circ}$ reported in [17] for the same vase sequence.

The intrinsics recovered from the optimized image invariants are shown in Table 1, which also shows the ground-truth values obtained with a classical calibration method [21] using an L-shape calibration pattern. It can be seen that the focal length $f$ and the $u_{0}$ coordinate of the principal point were both precisely estimated, while $v_{0}$ was not. This is due to the high uncertainty in the estimated coordinates of $\mathbf{v}_{x}$, which was far away from the image center for a camera looking at a direction close to the rotation axis. Note that, under the assumption of a natural camera, any error in the location of $\mathbf{v}_{x}$ in a direction parallel to $l_{s}$ will result in the same error in the location of the principal point. Fig. 14 shows the recovered positions and orientations of the rotating camera relative to the model,

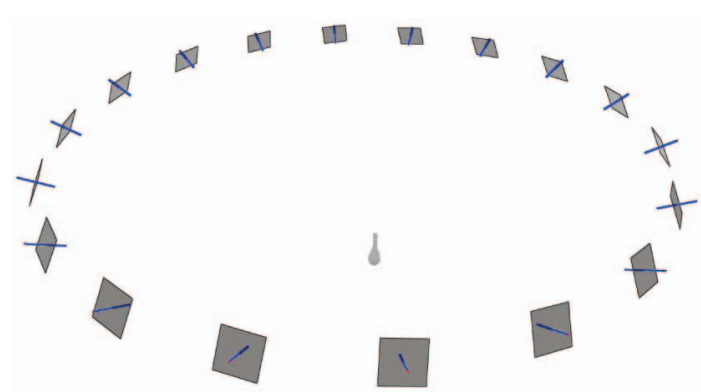

Fig. 14. The recovered camera positions and orientations for the vase sequence.

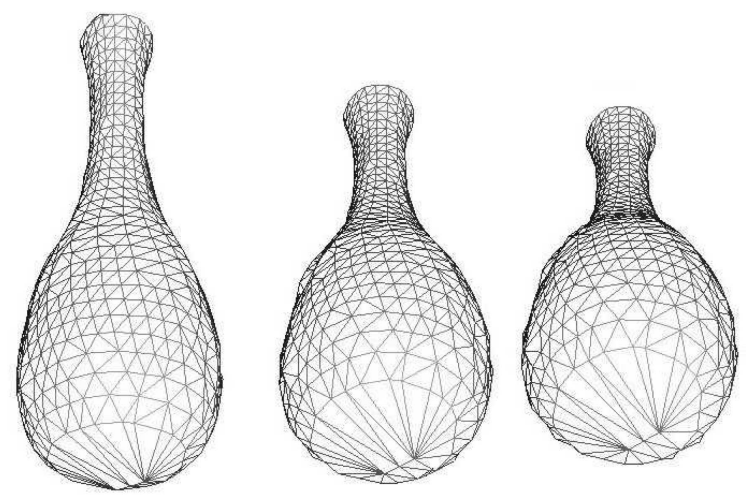

Fig. 15. Different views of the $3 \mathrm{D}$ model of the vase built from the estimated turntable motion.

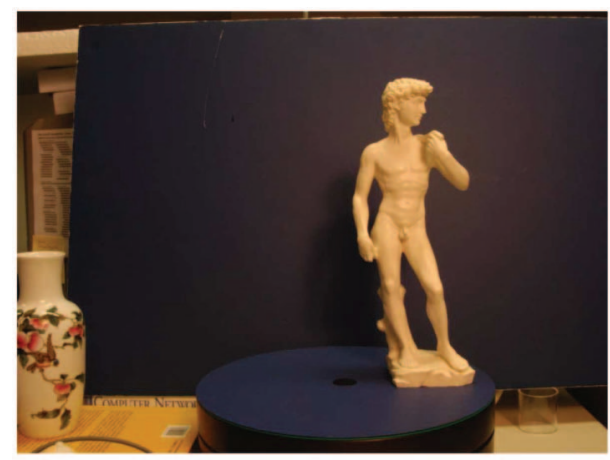

Fig. 16. An image from the David sequence.

and Fig. 15 shows three views of the 3D model reconstructed from the estimated motion.

The second sequence consists of 72 images of a David model with successive rotation angles of $5^{\circ}$ (see Fig. 16). The electronic turntable used has a resolution of $0.2^{\circ}$. Similar to the first experiment, after obtaining $\mathbf{l}_{\mathrm{s}}, \mathbf{v}_{x}$, and $\mathbf{l}_{\mathrm{h}}$ from the whole dense sequence, a subsequence consisting of 18 images with successive rotation angles of $20^{\circ}$ was extracted, and the same procedures for motion estimation, self-calibration, and euclidean reconstruction were applied to this subsequence.

Fig. 17 shows the histogram of the estimated values for the fixed scalar $\kappa$. Fig. 18 shows both the rotation angles obtained before and after the bundle adjustment. The RMS errors of the estimated rotation angles before and after the bundle adjustment are $0.170^{\circ}$ and $0.120^{\circ}$, respectively, which are better than the RMS error of $0.272^{\circ}$ achieved using the algorithm described in [17]. The calibration result 


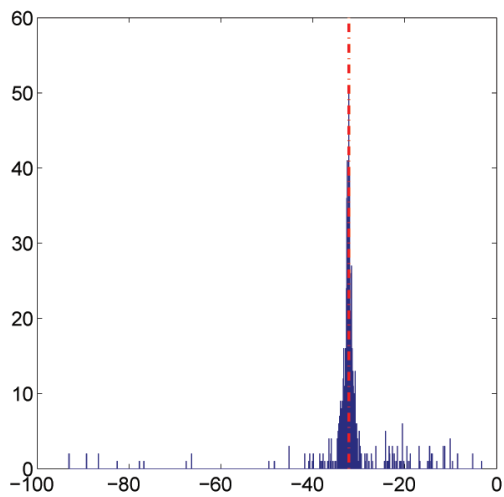

Fig. 17. David sequence: histogram of the estimated values for $\kappa$. The dashed line indicates the "best estimate" $\kappa_{0}$.

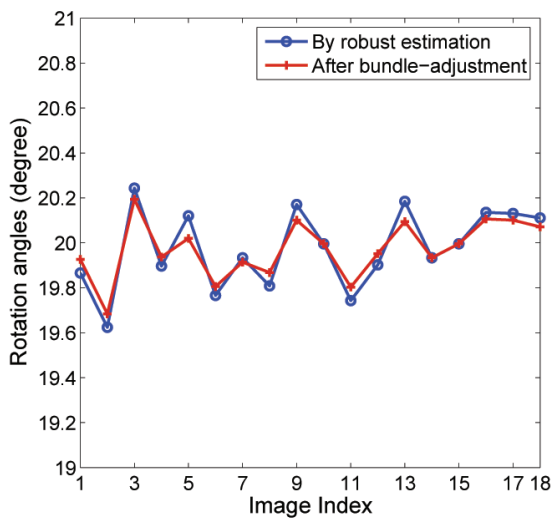

Fig. 18. David sequence: angles estimated using the robust method and those obtained after bundle adjustment. The RMS errors of the estimated rotation angles before and after the bundle adjustment are $0.170^{\circ}$ and $0.120^{\circ}$, respectively.

TABLE 2

Estimated and Ground-Truth Values for the Intrinsics of the David Sequence

\begin{tabular}{|c|ccc|}
\hline- & $f$ & $u_{0}$ & $v_{0}$ \\
\hline Ground-truth & 814.99 & 414.14 & 198.70 \\
Estimated value & 813.68 & 412.06 & 268.47 \\
Percentage error & $0.16 \%$ & $0.26 \%$ & $8.56 \%$ \\
\hline
\end{tabular}

is shown in Table 2. Fig. 19 shows the recovered positions and orientations of the rotating camera relative to the model. The reconstructed model is shown in Fig. 20, which reflects good qualities of our estimated parameters.

The third experiment was to test the robustness of the algorithm under different rotation angles. The dense sequence consisting of 72 vase images used in the first experiment was employed here. It provided 10 possible subsequences with $72,36,24,18,12,9,8,6$, 4, and 3 images, respectively. The corresponding rotation angle $\theta$ between adjacent images in these subsequences are $5^{\circ}, 10^{\circ}, 15^{\circ}, 20^{\circ}$, $30^{\circ}, 40^{\circ}, 45^{\circ}, 60^{\circ}, 90^{\circ}$, and $120^{\circ}$, respectively.

For each subsequence, the camera intrinsics and the rotation angles were computed in exactly the same way as in the first two experiments, except that the bundle adjustment step was omitted. The results are shown in Table 3. It can be seen that the camera calibration matrix can generally be estimated with high accuracy. However, the

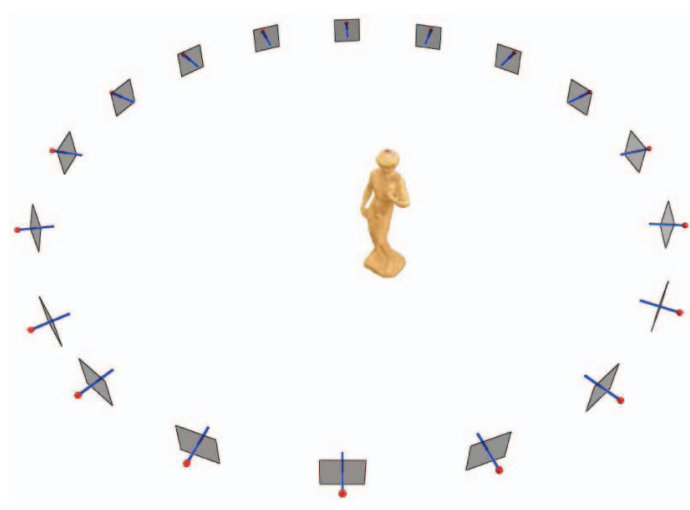

Fig. 19. The recovered camera positions and orientations for the David sequence.
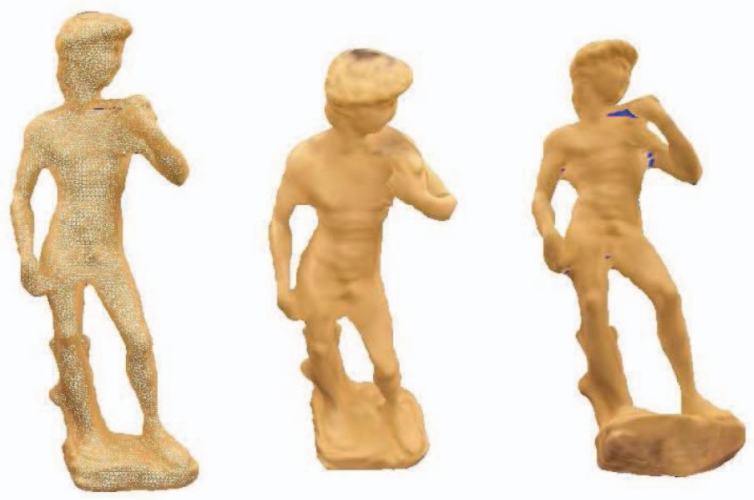

Fig. 20. Different views of the 3D model of David built from the estimated turntable motion.

TABLE 3

Calibration Results and the Estimated Angles under Different Rotation Angles

\begin{tabular}{|l|ccc|c|}
\hline$\theta$ & $f$ & $u_{0}$ & $v_{0}$ & rms err of angles \\
\hline $5^{\circ}$ & $2336.78(2.22 \%)$ & $350.87(0.34 \%)$ & $487.80(9.73 \%)$ & $0.08(1.57 \%)$ \\
\hline $10^{\circ}$ & $2339.88(2.09 \%)$ & $350.82(0.33 \%)$ & $487.80(9.73 \%)$ & $0.09(0.95 \%)$ \\
\hline $15^{\circ}$ & $2339.10(2.12 \%)$ & $350.83(0.33 \%)$ & $487.80(9.73 \%)$ & $0.16(1.05 \%)$ \\
\hline $20^{\circ}$ & $2345.88(1.84 \%)$ & $350.72(0.33 \%)$ & $487.81(9.73 \%)$ & $0.13(0.67 \%)$ \\
\hline $30^{\circ}$ & $2331.59(2.44 \%)$ & $350.95(0.34 \%)$ & $487.79(9.73 \%)$ & $0.22(0.74 \%)$ \\
\hline $40^{\circ}$ & $2364.02(1.08 \%)$ & $350.43(0.32 \%)$ & $487.84(9.73 \%)$ & $0.49(1.22 \%)$ \\
\hline $45^{\circ}$ & $2364.24(1.07 \%)$ & $350.42(0.32 \%)$ & $487.84(9.73 \%)$ & $0.41(0.90 \%)$ \\
\hline $60^{\circ}$ & $2402.15(0.52 \%)$ & $349.80(0.29 \%)$ & $487.91(9.73 \%)$ & $1.56(2.59 \%)$ \\
\hline $90^{\circ}$ & $2342.98(1.96 \%)$ & $350.77(0.33 \%)$ & $487.81(9.73 \%)$ & $19.72(21.91 \%)$ \\
\hline $120^{\circ}$ & $2266.31(5.17 \%)$ & $351.99(0.38 \%)$ & $487.68(9.72 \%)$ & $56.10(46.75 \%)$ \\
\hline
\end{tabular}

Columns 2-4 show the estimated intrinsic parameters. The values in brackets are the percentage errors relative to the ground-truth focal length. Column 5 shows the RMS errors of the estimated rotation angles. The values in brackets are the RMS errors of the estimated rotation angles relative to the ground-truth angles.

rotation angles could not be estimated accurately when the rotation angles are large. This is due to the fact that, under large rotation angles, the chance of having the degenerate case increases and, at the same time, the number of epipoles available for robust estimation of the angles decreases. Hence, the accuracy of the estimated rotation angles decreases accordingly.

\section{Discussions and Conclusions}

In this paper, a simple and practical approach has been introduced to recover the camera intrinsics and the relative 
rotation angles from silhouettes in a turntable sequence. A special parameterization for the imaged circular points of the turntable plane in terms of the image invariants and a fixed scalar is derived. This allows the camera intrinsics to be obtained directly from the estimated image invariants. Besides, a robust method is developed to estimate the rotation angles. This method does not depend on the knowledge of the camera intrinsics, and can handle the degenerate case in which the fundamental matrix is not recoverable. The proposed algorithm takes an uncalibrated turntable sequence as input, and self-calibration is carried out to achieve a euclidean reconstruction. Experiments on two image sequences showed that both the camera intrinsics and the rotation angles could be estimated with a high precision, and convincing 3D models have been constructed using these estimated parameters.

\section{REFERENCES}

[1] R. Szeliki, "Shape from Rotation," Proc. IEEE Int'l Conf. Computer Vision and Pattern Recognition, pp. 625-630, 1991.

[2] W. Niem, "Robust and Fast Modeling of 3D Natural Objects from Multiple Views," SPIE Proc.: Image and Video Processing II, vol. 2182, pp. 388-397, Feb. 1994.

[3] A.W. Fitzgibbon, G. Cross, and A. Zisserman, "Automatic 3D Model Construction for Turn-Table Sequences," Proc. European Workshop 3D Structure from Multiple Images of Large-Scale Environments, pp. 155-170, 1998.

[4] G. Jiang, H.T. Tsui, L. Quan, and A. Zisserman, "Single Axis Geometry by Fitting Conics," Proc. Seventh European Conf. Computer Vision, pp. 482-488, 2002.

[5] G. Jiang, L. Quan, and H. Tsui, "Circular Motion Geometry by Minimal 2 Points in 4 Images," Proc. Ninth IEEE Int'l Conf. Computer Vision, pp. 221-227, 2003.

[6] R. Cipolla and P.J. Giblin, Visual Motion of Curves and Surfaces. Cambridge Univ. Press, 1999.

[7] R. Cipolla, K. Åström, and P. Giblin, "Motion from the Frontier of Curved Surfaces," Proc. Fifth IEEE Int'l Conf. Computer Vision, pp. 269-275, June 1995.

[8] J.H. Rieger, "3 Dimensional Motion from Fixed Points of a Deforming Profile Curve," Optics Letters, vol. 3, no. 11, pp. 123125, 1986.

[9] J. Porrill and S.B. Pollard, "Curve Matching and Stereo Calibration," Image and Vision Computing, vol. 9, no. 1, pp. 45-50, Feb. 1991.

[10] K. Åström, R. Cipolla, and P. Giblin, "Generalised Epipolar Constraints," Int'l J. Computer Vision, vol. 33, no. 1, pp. 51-72, Sept. 1999.

[11] T. Joshi, N. Ahuja, and J. Ponce, "Structure and Motion Estimation from Dynamic Silhouettes under Perspective Projection," Int'l J. Computer Vision, vol. 31, no. 1, pp. 31-50, Feb. 1999.

[12] S. Sinha, M. Pollefeys, and L. McMillan, "Camera Network Calibration from Dynamic Silhouettes," Proc. IEEE Int'l Conf. Computer Vision and Pattern Recognition, pp. 195-202, June-July 2004.

[13] P.R.S. Mendonça, K.-Y.K. Wong, and R. Cipolla, "Camera Pose Estimation and Reconstruction from Image Profiles under Circular Motion," Proc. Sixth European Conf. Computer Vision, vol. II, pp. 864-877, 2000.

[14] K.-Y.K. Wong and R. Cipolla, "Structure and Motion from Silhouettes," Proc. Eighth IEEE Int'l Conf. Computer Vision, vol. II, pp. 217-222, July 2001.

[15] C. Hernández, F. Schmitt, and R. Cipolla, "Silhouette Coherence for Camera Calibration under Circular Motion," IEEE Trans. Pattern Analysis and Machine Intelligence, vol. 29, no. 2, pp. 343-349, Feb. 2007.

[16] Y. Furukawa, A. Sethi, J. Ponce, and D.J. Kriegman, "Robust Structure and Motion from Outlines of Smooth Curved Surfaces," IEEE Trans. Pattern Analysis and Machine Intelligence, vol. 28, no. 2, pp. 302-315, Feb. 2006.
[17] P.R.S. Mendonça, K.-Y.K. Wong, and R. Cipolla, "Epipolar Geometry from Profiles under Circular Motion," IEEE Trans. Pattern Analysis and Machine Intelligence, vol. 23, no. 6, pp. 604-616, June 2001.

[18] K.-Y.K. Wong and R. Cipolla, "Reconstruction of Sculpture from Its Profiles with Unknown Camera Positions," IEEE Trans. Image Processing, vol. 13, no. 3, pp. 381-389, Mar. 2004.

[19] H. Zhang, G. Zhang, and K.-Y.K. Wong, "Auto-Calibration and Motion Recovery from Silhouettes for Turntable Sequences," Proc. British Machine Vision Conf., vol. I, pp. 79-88, Sept. 2005.

[20] K.-Y.K. Wong, P.R.S. Mendonça, and R. Cipolla, "Camera Calibration from Surfaces of Revolution," IEEE Trans. Pattern Analysis and Machine Intelligence, vol. 25, no. 2, pp. 147-161, Feb. 2003.

[21] R. Hartley and A. Zisserman, Multiple View Geometry in Computer Vision. Cambridge Univ. Press, 2004.

[22] T. Vieville and D. Lingrand, "Using Singular Displacements for Uncalibrated Monocular Visual Systems," Proc. Fourth European Conf. Computer Vision, pp. 207-216, Apr. 1996.

[23] J. Gentle, Numerical Linear Algebra for Applications in Statistics. Springer-Verlag, 1998.

[24] G. Zhang, H. Zhang, and K.-Y.K. Wong, "1D Camera Geometry and Its Application to Circular Motion Estimation," Proc. British Machine Vision Conf., vol. I, pp. 67-76, Sept. 2006.

[25] J.G. Semple and G.T. Kneebone, Algebraic Projective Geometry. Clarendon Press, 1998.

[26] R. Cipolla and A. Blake, "The Dynamic Analysis of Apparent Contours," Proc. Third IEEE Int'l Conf. Computer Vision, pp. 616623, Dec. 1990.

[27] R. Cipolla and A. Blake, "Surface Shape from the Deformation of Apparent Contours," Int'l J. Computer Vision, vol. 9, no. 2, pp. 83112, Nov. 1992.

[28] C. Liang and K.-Y.K. Wong, "Complex 3D Shape Recovery Using a Dual-Space Approach," Proc. IEEE Int'l Conf. Computer Vision and Pattern Recognition, vol. 2, pp. 878-884, June 2005.

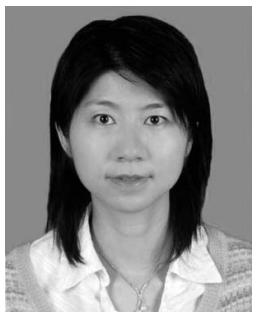

Hui Zhang received the BEng and MEng degrees (with honors) in computer science and engineering from Zhejiang University in 2002 and the $\mathrm{PhD}$ degree in computer vision from the University of Hong Kong in 2007. She is currently with the Department of Computer Science and Technology at United International College as an assistant professor. Her research interests include computer graphics, computer vision, and image processing, in particular camera calibration, motion estimation from image sequences, and model reconstruction and representation. She is a member of the IEEE.

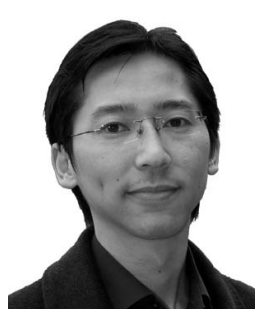

Kwan-Yee K. Wong received the BEng degree (with first-class honors) in computer engineering from the Chinese University of Hong Kong in 1998 and the MPhil and PhD degrees in computer vision (information engineering) from the University of Cambridge in 2000 and 2001, respectively. Since 2001 , he has been with the Department of Computer Science at the University of Hong Kong, where he is currently an associate professor. His research interests include computer vision and image processing, in particular camera calibration, motion tracking, model reconstruction and representation, and motion estimation from image sequences. He is a member of the IEEE.

$>$ For more information on this or any other computing topic, please visit our Digital Library at www.computer.org/publications/dlib. 